\title{
Occurrence of neoxanthin and lutein epoxide cycle in parasitic Cuscuta species
}

\author{
Jerzy Kruk ${ }^{\bowtie}$ and Renata Szymańska \\ Department of Plant Physiology and Biochemistry, Faculty of Biochemistry, Biophysics and Biotechnology, \\ Jagiellonian University, Kraków, Poland
}

Received: 12 November, 2007; revised: 07 January, 2008; accepted: 15 January, 2008

available on-line: 24 January, 2008

\begin{abstract}
In the present study, xanthophyll composition of eight parasitic Cuscuta species under different light conditions was investigated. Neoxanthin was not detected in four of the eight species examined, while in others it occurred at the level of several percent of total xanthophylls. In C. gronovii and C. lupuliformis it was additionally found that the neoxanthin content was considerably stimulated by strong light. In dark-adapted plants, lutein epoxide level amounted to $10-22 \%$ of total xanthophylls in only three species, the highest being for $C$. lupuliformis, while in others it was below $3 \%$, indicating that the lutein epoxide cycle is limited to only certain $\mathrm{Cus}$ cuta species. The obtained data also indicate that the presence of the lutein epoxide cycle and of neoxanthin is independent and variable among the Cuscuta species. The xanthophyll cycle carotenoids violaxanthin, antheraxanthin and zeaxanthin were identified in all the examined species and occurred at the level found in other higher plants. The xanthophyll and lutein epoxide cycle pigments showed typical response to high light stress. The obtained results also suggest that the ability of higher plants to synthesize lutein epoxide probably does not depend on the substrate specificity of zeaxanthin epoxidase but on the availability of lutein for the enzyme.
\end{abstract}

Keywords: Cuscuta, lutein epoxide cycle, neoxanthin, violaxanthin de-epoxidase, xanthophyll cycle, zeaxanthin epoxidase

\section{INTRODUCTION}

The light-dependent, reversible changes of xanthophyll pigments, violaxanthin, antheraxanthin and zeaxanthin, called the xanthophyll cycle, are the main photoprotective mechanism of the photosynthetic apparatus in higher plants (Demming-Adams \& Adams, 1992; Latowski et al., 2004b). The conversion of violaxanthin into zeaxanthin is catalyzed by the lumen enzyme violaxanthin de-epoxidase that requires ascorbate as a cofactor and is activated by low $\mathrm{pH}$ of the lumen during high-light illumination. The activity of the enzyme is stimulated by the presence of membrane lipids forming hexagonal phases, such as monogalactosyldiacylglycerol (Latowski et al., 2002) or others (Latowski et al., 2004a). Zeaxan- thin epoxidase, the enzyme catalyzing the reverse reaction, has not been isolated so far, but its aminoacid sequence is known for many plant species, and it was found that it contains FAD as a cofactor and requires $\mathrm{NADPH}$, molecular oxygen and ferredoxin for activity (Latowski et al., 2004b). Both enzymes belong to the group of lipocalins (Grzyb et al., 2006).

In some diatoms, a cycle has been identified where diadinoxanthin is converted to diatoxanthin (Latowski et al., 2004b). In this case, the epoxidation proceeds only to the stage of diatoxanthin containing one epoxy group.

In recent years, lutein epoxide cycle has been identified in holoparasitic Cuscuta reflexa (Bungard et al., 1999), hemiparasitic plants (Matsubara et al., 2001; 2003), Quercus species (Garcia-Plazaola et al., 2002),

${ }^{\square}$ Corresponding author: Jerzy Kruk, Department of Plant Physiology and Biochemistry, Faculty of Biochemistry, Biophysics and Biotechnology, Jagiellonian University, Gronostajowa 7, 30-387 Kraków, Poland; tel.: (48) 12 664 6361; fax: (48) 12 664 6902; e-mail: jkruk@mol.uj.edu.pl

Abbreviations: A, antheraxanthin; Car, carotenoids; Chl, chlorophyll; L, lutein; LHC, light-harvesting complex; Lx, lutein epoxide; N, neoxanthin; V, violaxanthin; Z, zeaxanthin. 
a tropical tree Inga sapindoides, and others (GarciaPlazaola et al., 2007) where reversible light-induced changes in the level of lutein epoxide and lutein were found. This cycle was suggested, similarly to the classical xanthophyll cycle, to have a photoprotective function in these plants (Garcia-Plazaola et al., 2007). Moreover, it has been shown recently that accumulation of lutein epoxide can increase the light harvesting efficiency in the antennae of photosystem II (Garcia-Plazaola et al., 2007). The content of lutein epoxide in dark-adapted plants showing the lutein epoxide cycle is usually lower than that of violaxanthin and it was found to comprise $18 \%$ of total xanthophylls in C. reflexa (Snyder et al., 2005), 15-18\% in the parasitic Amyema miquelii (Matsubara et al., 2001; 2003), between 1 and $11 \%$ in other members of the parasitic Loranthaceae family (Matsubara et al., 2003), between 0 and $5 \%$ of the Viscaceae family (Matsubara et al., 2003) and other investigated parasitic plants (Matsubara et al., 2003), and up to $10 \%$ in different Quercus species (Garcia-Plazaola et al., 2002). Recently, it was shown that the tropical tree legume Inga sp. contains extraordinary high levels of lutein epoxide accounting for $30 \%$ of total xanthophylls and a threefold higher amount than that of violaxanthin in dark-adapted leaves (Matsubara et al., 2005). In this species, the lutein epoxide cycle was suggested to play a role in photoacclimation and shade adaptation (Matsubara et al., 2007). In leaves of other green plants, lutein epoxide is frequently found but only in minor amounts (Goodwin, 1980; Lichtenthaler, 1987), therefore the occurrence and significance of the lutein epoxide cycle seems to be limited to only certain plant species.

The question arises as to the nature of the enzymes engaged in the lutein epoxide cycle. It was found that isolated violaxanthin de-epoxidase catalyzes both violaxanthin and lutein epoxide de-epoxidation (Yamamoto \& Higashi, 1978; Grotz et al., 1999; Matsubara et al., 2003), thus it may be concluded that the same enzyme is involved in de-epoxidation reactions of both violaxanthin and lutein epoxide cycles. However, in the case of the epoxidation reaction it is not known if there is only one or two enzymes catalyzing epoxidation of zeaxanthin and lutein. The presence of lutein epoxide in only some plant species, together with the universal occurrence of lutein, is taken as an argument that two separate enzymes of different specificity are involved in both cycles. Such a view is also supported by a report that cloned epoxidase from Capsicum sp. was specific for zeaxanthin and not for lutein (Bouvier et al., 1996). On the other hand, there are many plant species known to accumulate high lutein epoxide levels in flowers and fruits (Goodwin, 1980), but not in leaves. Moreover, leaves of etiolated plants, in contrast to green leaves, show relatively high levels of lutein epoxide, like for example Pinus silvestris (13.5\% of total xanthophylls) (Schoefs \& Franck, 1988) or cabbage varieties (7.7$15 \%$ of total xanthophylls) (Kruk, 2005). The level of lutein epoxide in partially etiolated vegetative buds of woody plants was also found to be increased as compared to green leaves (Garcia-Plazaola et al., 2004). These data, in turn, might suggest that there is one enzyme for both lutein and zeaxanthin epoxidation, but the increased level of lutein epoxide in etiolated leaves is due to a higher availability of lutein for the epoxidase. Moreover, genetic approach suggests that only one epoxidase enzyme exists in a given plant species, since only one gene has been detected for zeaxanthin epoxidase in all the species examined so far (Matsubara et al., 2003).

The photosynthetic pigment composition of Cuscuta reflexa, the most thoroughly characterized species of the holoparasitic Cuscuta genus (Hibberd et al., 1998), was exceptional in the complete absence of neoxanthin in this parasitic plant and this was the first example of a higher plant lacking this carotenoid (Bungard et al., 1999) which is usually present at $5-13 \%$ of total carotenoids (Lichtenthaler, 1987). It had also been suggested that lutein epoxide replaces neoxanthin in the LHC of $C$. reflexa (Bungard et al., 1999), however, it was later shown (Snyder et al., 2004) that 9-cis-violaxanthin is the carotenoid replacing neoxanthin in this species. Other parasitic plants examined so far (Matsubara et al., 2001; 2003) showed normal level of neoxanthin regardless of the content of lutein epoxide. However, no other Cuscuta species have been examined in this respect to date.

In the present report we examined the photosynthetic pigment composition of eight Cuscuta species and the response of the composition of xanthophylls to high light stress. The obtained results show that the examined species differ considerably in the presence of lutein epoxide and neoxanthin content.

\section{MATERIALS AND METHODS}

Plant material. Cuscuta epithymum (growing on Lotus corniculatus), C. europaea (growing on Urtica dioica) and C. lupuliformis (growing on Rubus sp.) were collected from natural standings around Kraków, while the other species were a kind gift of Karin Krupinska from Kiel University (Germany) and were grown in a greenhouse on Pelargonium zonale under shade. All the Cuscuta species were analyzed in late August / early September. For xanthophyll cycle measurements, shoots were dark-adapted for $16 \mathrm{~h}$ before the analysis or alternatively illuminated for $30 \mathrm{~min}$ with high-light of $1500 \mu \mathrm{mol} / \mathrm{m}^{2}$ per s. For all the Cuscuta species, only $10 \mathrm{~cm}$ long tip shoots were taken for analysis. The tips of the shoots were detached from the plant and their cut 
ends were placed in tap water during dark-adaptation and high light illumination.

Pigments analysis. Routinely, 100-150 mg of Cuscuta shoots was ground in a mortar with 1.5 $\mathrm{ml}$ of cold HPLC solvent (acetonitrile/methanol/water, $72: 8: 1$, by vol.). The extract was transferred to an Eppendorf tube, shortly centrifuged $(60 \mathrm{~s})$ on a benchtop centrifuge $(10000 \times g)$ and analysed by HPLC.

The HPLC measurements were performed using $100 \mu \mathrm{l}$ loop, Jasco PU-980 pump and UV-VIS detector system UV-970 with the detection wavelength of $440 \mathrm{~nm}$, Teknokroma (Barcelona, Spain) C18 reverse-phase column (Nucleosil 100, $250 \times 4 \mathrm{~mm}, 5$ $\mu \mathrm{m})$, isocratic solvent system - acetonitrile/methanol/water (72:8:1, by vol.) at the flow rate of 2 or $1.5 \mathrm{ml} / \mathrm{min}$.

Chlorophylls and total carotenoids were determined spectrophotometrically according to the method of Lichtenthaler (1987).

\section{RESULTS}

\section{Neoxanthin analysis}

The analysis of neoxanthin content in eight Cuscuta species revealed that, apart from C. reflexa also some other species were deficient in this carotenoid. Among the investigated Cuscuta species, neoxanthin could not be identified in C. epithymum, C. japonica and C. reflexa, only traces were found in C. europaea, while the other species showed a low but evident presence of neoxanthin, reaching the highest content in C. campestris (Fig. 1, Table 1). The identity of neoxanthin in the investigated species was confirmed by absorption spectra and the effect of $\mathrm{HCl}$ on the absorption spectrum (not shown). Interestingly, the level of neoxanthin was clearly stimulated in the case of $C$. gronovii and C. lupuliformis (Fig. 2) under high light conditions. The increase in neoxanthin level is most probably at the expense of violaxanthin which is its biosynthetic precursor.

\section{Lutein epoxide cycle}

The lutein epoxide content varied considerably among the investigated species. In dark-adapted plants, lutein epoxide level was the highest in C. lupuliformis, followed by C. reflexa and C. japonica (Table 1, Fig. 1), where its content was between 10 and $22 \%$ of total xanthophylls. In the case of C. $l u$ puliformis, the level of lutein epoxide even exceeded that of violaxanthin. On the other hand, the content of lutein epoxide in the other species was considerably lower (Table 1) but detectable and resembled

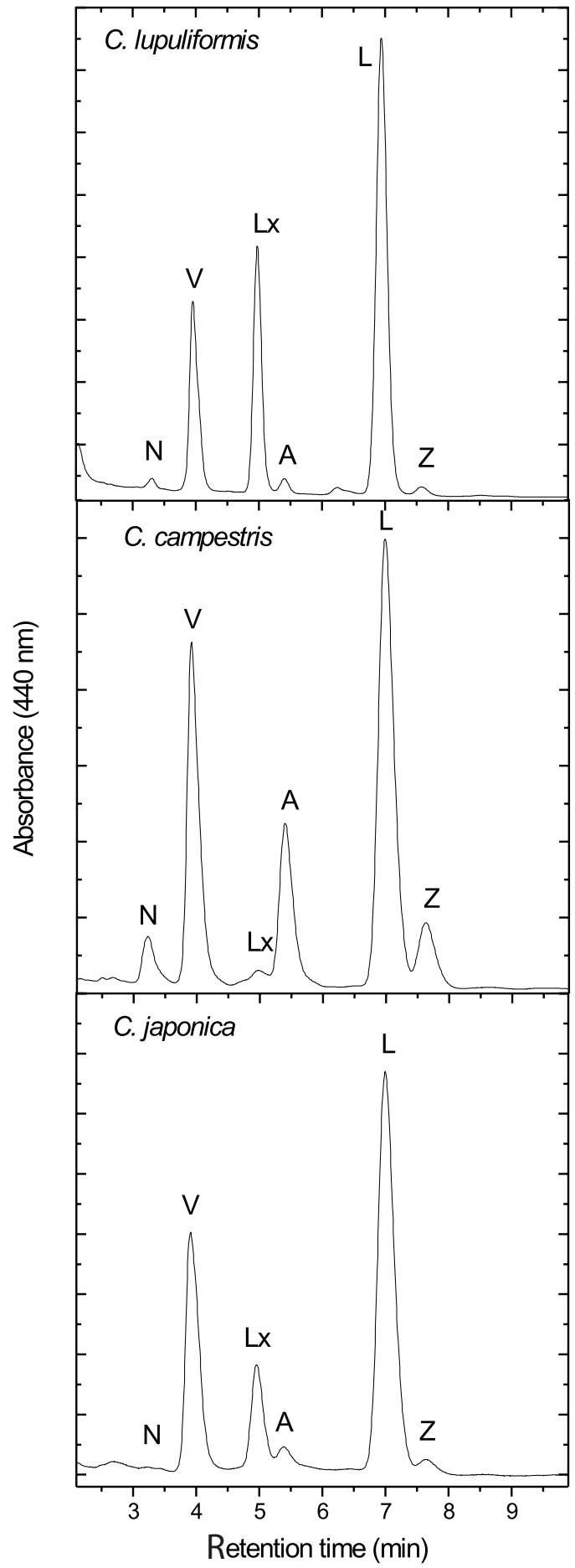

Figure 1. HPLC chromatograms of xanthophylls in dark adapted shoots of different Cuscuta species.

Details are given in Materials and Methods. A, antheraxanthin; L, lutein; Lx, lutein epoxide, N, neoxanthin; V, violaxanthin.

that in most of green plants. In order to find out if the lutein epoxide cycle is functional in the four Cuscuta species with the highest lutein epoxide content, dark-adapted shoots of these species were exposed to high light stress. It was found that under 
Table 1. Composition of xanthophylls (\% total), ratio of chlorophylls and total carotenoids to chlorophylls ratio in dark adapted Cuscuta species.

A, antheraxanthin; L, lutein; Lx, lutein epoxide, N, neoxanthin; V, violaxanthin; Z, zeaxanthin; Chl, chlorophyll; Car, total carotenoids. $N=3 \pm$ S.E. (three shoots were simultaneously dark-incubated and afterwards independently analyzed).

\begin{tabular}{lllllllll}
\hline Cuscuta species & $\mathrm{N}$ & $\mathrm{V}$ & $\mathrm{Lx}$ & $\mathrm{A}$ & $\mathrm{L}$ & $\mathrm{Z}$ & Chl a/b (w/w) & $\begin{array}{l}\text { Car/Chls } \\
(\mathrm{w} / \mathrm{w})\end{array}$ \\
\hline C. campestris & $4.0 \pm 0.3$ & $28.5 \pm 2.1$ & $0.5 \pm 0.1$ & $14.3 \pm 1.0$ & $47.0 \pm 2.3$ & $5.6 \pm 0.4$ & $3.62 \pm 0.25$ & $0.54 \pm 0.06$ \\
C. epithymum & 0 & $23.1 \pm 1.9$ & $0.6 \pm 0.2$ & $17.6 \pm 1.4$ & $50.0 \pm 2.1$ & $8.8 \pm 0.7$ & $3.78 \pm 0.08$ & $0.89 \pm 0.15$ \\
C. europaea & $\leq 0.1$ & $23.2 \pm 2.5$ & $1.8 \pm 0.4$ & $7.8 \pm 0.9$ & $62.4 \pm 3.6$ & $4.7 \pm 0.6$ & $2.95 \pm 0.04$ & $0.60 \pm 0.08$ \\
C. gronovii & $3.1 \pm 0.2$ & $44.4 \pm 2.1$ & $0.9 \pm 0.2$ & $7.7 \pm 2.4$ & $43.2 \pm 2.0$ & $0.6 \pm 0.3$ & $3.98 \pm 0.11$ & $0.63 \pm 0.13$ \\
C. japonica & 0 & $28.9 \pm 2.1$ & $10.2 \pm 0.7$ & $1.3 \pm 0.4$ & $58.5 \pm 3.2$ & $1.0 \pm 0.6$ & $3.00 \pm 0.03$ & $0.47 \pm 0.05$ \\
C. lupuliformis & $0.9 \pm 0.2$ & $18.4 \pm 0.9$ & $22.2 \pm 0.3$ & $1.1 \pm 0.3$ & $56.0 \pm 1.5$ & $0.9 \pm 0.2$ & $2.78 \pm 0.05$ & $0.42 \pm 0.06$ \\
C. platyloba & $0.6 \pm 0.1$ & $50.0 \pm 2.6$ & $2.8 \pm 0.2$ & $4.1 \pm 1.2$ & $41.2 \pm 2.1$ & $1.3 \pm 0.3$ & $2.27 \pm 0.17$ & $0.51 \pm 0.10$ \\
C. reflexa & 0 & $44.6 \pm 2.3$ & $18.1 \pm 0.5$ & $0.2 \pm 0.1$ & $36.3 \pm 1.4$ & $0.7 \pm 0.4$ & $2.80 \pm 0.04$ & $0.47 \pm 0.07$ \\
\hline
\end{tabular}

such conditions, the content of lutein epoxide clearly decreased in all four species examined (Fig. 3). The content of lutein epoxide recovered to the original level within $16 \mathrm{~h}$ of dark adaptation (not shown). These observations indicate the activity of the lutein epoxide cycle in the investigated species.

\section{Xanthophyll cycle}

The analysis of the xanthophyll cycle pigments (violaxanthin, antheraxanthin, zeaxanthin) of dark adapted shoots of Cuscuta species showed (Table 1, Figs. 4 and 5) that in the Cuscuta species showing only trace amounts of lutein epoxide, the level of antheraxanthin and zeaxanthin remains relatively high (Fig. 4). The level of both of these xanthophylls did not change significantly even when the dark adaptation was extended from 16 to 24 hours (not shown). This suggests that a fraction of antheraxanthin and zeaxanthin is not available for the epoxidase in these species in contrast to the species with a high level of lutein epoxide. Under high light exposure, violaxanthin is convert-

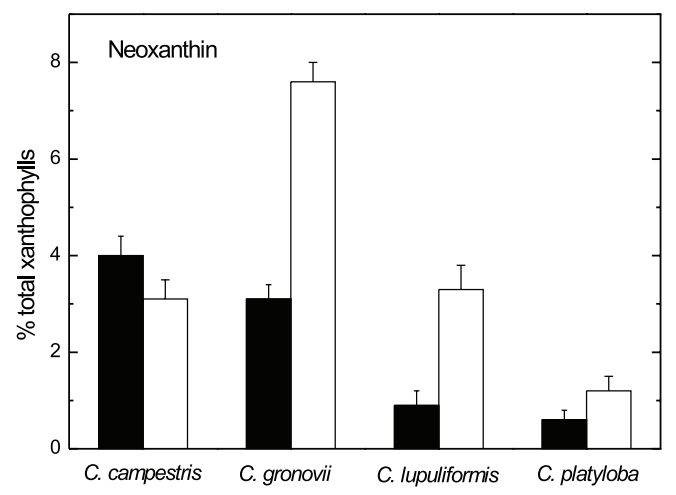

Figure 2. Neoxanthin content in selected Cuscuta species. Dark-adapted shoots (black bars), after $30 \mathrm{~min}$ of high light illumination (white bars). Details are given in Table 1 . ed to zeaxanthin via antheraxanthin (Figs. 4 and 5 ), indicating the activity of the xanthophyll cycle in the investigated species. However, the observed extent of violaxanthin conversion is different among the species. It is most pronounced for $C$. platyloba, C. reflexa, C. japonica, C. epithymum and C. gronovii (Figs. 4 and 5). In the case of C. campestris, C. europaea and C. lupuliformis the extent of violaxanthin de-epoxidation was only $23-30 \%$ and this might be related to a lower epoxidase activity or lower violaxanthin availability for the enzyme in these species. Interestingly, the highest extent of violaxanthin and antheraxanthin de-epoxidation under high light is observed for the Cuscuta species showing also high levels of lutein epoxide $(C$. japonica, C. platyloba, C. reflexa). This suggests that the availability of carotenoids for the de-epoxidation reaction is the highest in these species. The total content of xanthophyll pigments before and after illumination was the same within the experimental error for most of the species examined (Fig. 6).

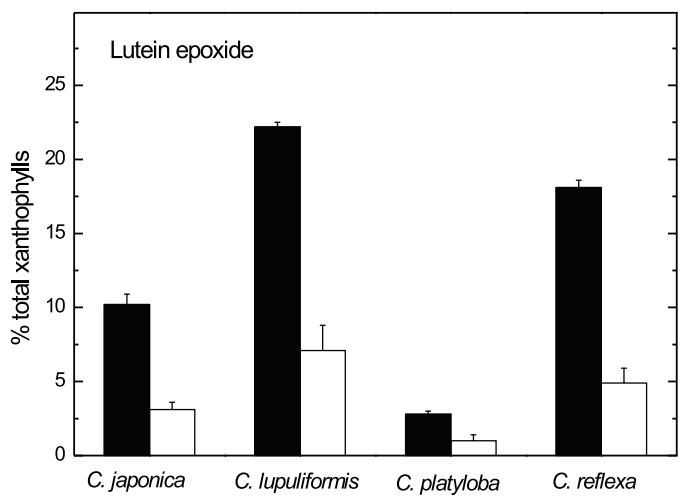

Figure 3. Lutein epoxide content in selected Cuscuta species.

Dark-adapted shoots (black bars), after $30 \mathrm{~min}$ of high light illumination (white bars). Details are given in Table 1 . 

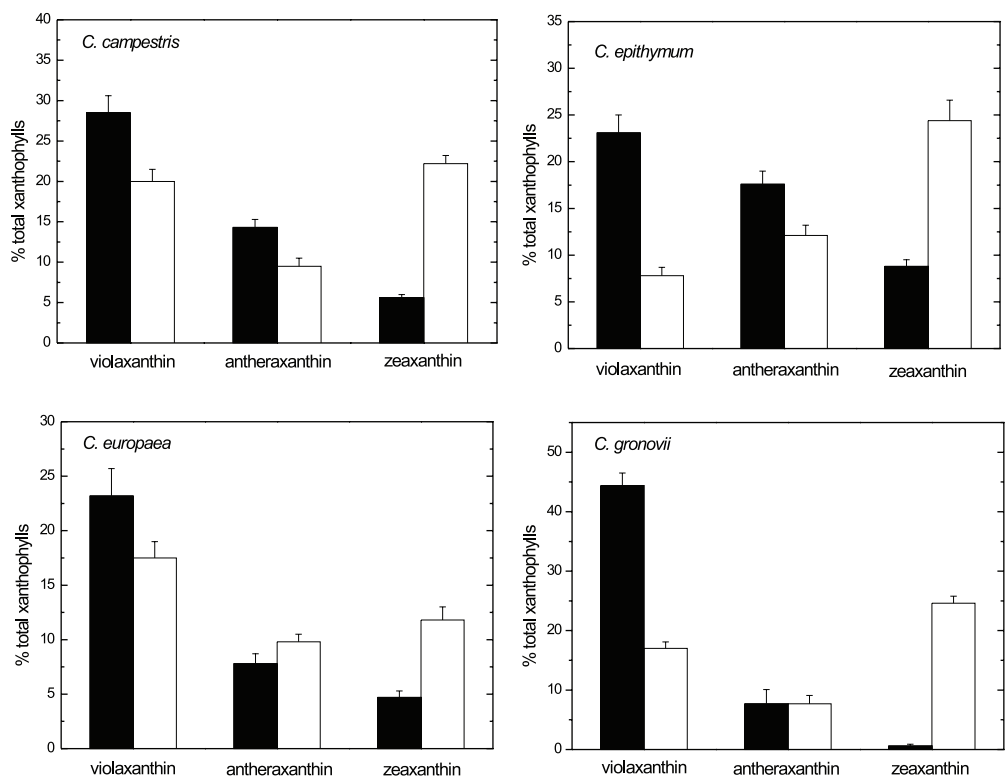

Figure 4. The content of xanthophyll cycle pigments in different Cuscuta species.

Dark-adapted shoots (black bars), after $30 \mathrm{~min}$ of high light illumination (white bars). Details are given in Table 1 .

The ratio of chlorophylls varied among the investigated species (Table 1), but within values reported for higher plants (Lichtenthaler, 1987). On the other hand, the ratio of total carotenoids to chlorophylls in all the Cuscuta species exceeds twofold or more the values typically found for chloroplasts of higher plants (0.25) (Lichtenthaler, 1987) and this indicates that a fraction of xanthophylls in the investigated Cuscuta species is outside the LHC complexes or that some xanthophylls-binding complexes are chlorophyll-deficient.

\section{DISCUSSION}

The obtained results show that the investigated Cuscuta species differ considerably in the presence of neoxanthin and of the lutein epoxide cycle. The only species of this genus investigated so far, C. reflexa, was different from other plants in lacking neoxanthin and having a high level of lutein epoxide (Bungard et al., 1999), which was confirmed in the present study. The carotenoid composition of this species was also unusual by the presence of 9-cis-violaxanthin replacing neoxanthin
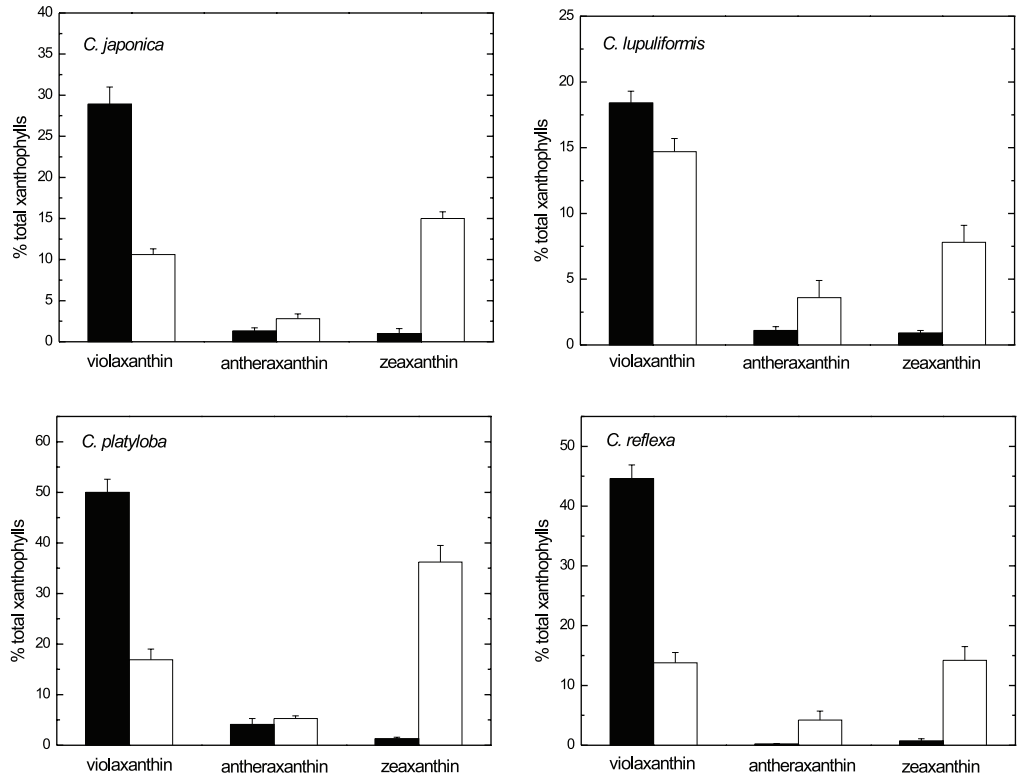

Figure 5. The content of xanthophyll cycle pigments in different Cuscuta species.

Dark-adapted shoots (black bars), after $30 \mathrm{~min}$ of high light illumination (white bars). Details are given in Table 1. 


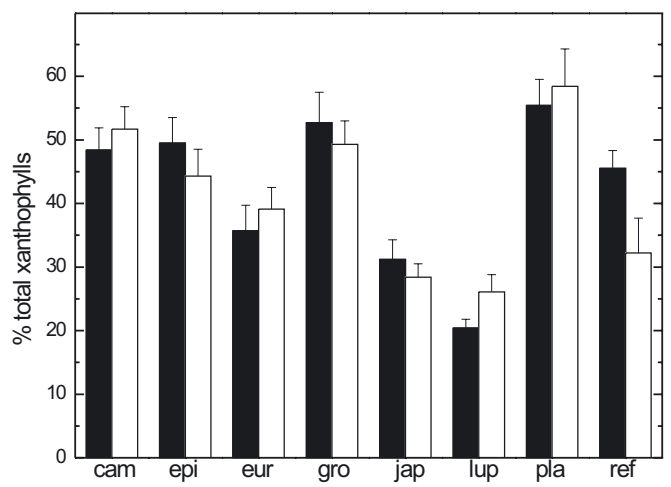

Figure 6. The sum of xanthophyll cycle pigments in different Cuscuta species.

Dark-adapted shoots (black bars), after $30 \mathrm{~min}$ of high light illumination (white bars). Details are given in Table 1 .

in LHCb complexes (Snyder et al., 2004). Unfortunately, the chromatographic system used in the present study was not able to resolve violaxanthin isomers and the content of 9-cis-violaxanthin could not be quantified in the other Cuscuta species. Although the proportion of carotenoids to chlorophyll found in our study for all the species examined was higher than that reported for green plants, it was lower compared to some species investigated earlier in this respect, such as C. gronovii, C. platyloba and C. campestris (van der Kooij et al., 2000; 2005). However, it is known that the relative pigment composition in Cuscuta species strongly depends on different conditions such as tissue age, growth conditions, host type, tissue starvation, etc. (van der Kooij et al., 2000; 2005).

\section{Neoxanthin analysis}

Our data revealed that apart from C. reflexa, also some other Cuscuta species are deficient in neoxanthin, being unique among higher plants in this respect. However, the lack of neoxanthin is not common to all Cuscuta species. The absence of neoxanthin does not correlate with the presence of the lutein epoxide cycle. Neoxanthin is a component of the LHC and it has been found that in C. reflexa, neoxanthin is replaced by 9-cis-violaxanthin in the complex (Snyder et al., 2004). Probably, a similar replacement also takes place in the other neoxanthindeficient species.

The inability to synthesize neoxanthin in several of the Cuscuta species investigated could be due to a deletion of the neoxanthin synthase gene or a lack of its expression. It is known that in the course of evolution Cuscuta species have lost some chloroplast-encoded genes due to parasitism (Berg et al., 2004). If neoxanthin synthase gene is missing in some of the Cuscuta species, this would be the first example of a loss of a nuclear gene as a result of parasitism. In the Cuscuta species with a low but detectable neoxanthin content (C. platyloba, C. lupuliformis) the gene must be present but its activity is apparently low. Interestingly, in C. gronovii and C. lupuliformis the neoxanthin level is stimulated 2.5 and 4-fold under strong light, respectively. The content of neoxanthin in high-light treated C. gronovii $(8 \%)$ reached the neoxanthin level observed in green plants (5-13\%) (Lichtenthaler, 1987). This increase is most probably due to the enhanced expression of neoxanthin synthase.

\section{Lutein epoxide cycle}

The investigated Cuscuta species differ considerably in the content of lutein epoxide in dark adapted plants, from the level below $1 \%$ of total xanthophylls to about $20 \%$ in C. reflexa and C. lupuliformis. Among the species of a single genus investigated so far, like Quercus (Garcia-Plazaola et al., 2004) or some parasitic plants (Matsubara et al., 2003), great variations of the lutein epoxide content were also observed. Lutein epoxide cycle is considered as an additional photoprotective mechanism, similar to the xanthophyll cycle (Garcia-Plazaola et al., 2007). Its function in shade adaptation for tree leaves has also been demonstrated (Garcia-Plazaola et al., 2007). The Cuscuta species in our study (C. lupuliformis, C. epithymum, C. europaea) collected from natural habitats grew under similar conditions (unshaded) and showed considerable differences regarding the activity of the lutein epoxide cycle. Thus, in the case of Cuscuta species the presence of the lutein epoxide cycle can be considered as an additional photoprotective mechanism against excess light conditions, but its presence is limited to some species only.

The considerable differences in the activity of the lutein epoxide cycle are interesting in terms of the substrate specificity of zeaxanthin epoxidase. In the available genome databases only one gene of zeaxanthin epoxidase can be found in a given plant species, meaning that it is hardly probable that separate enzymes exist for epoxidation of zeaxanthin and lutein in the same species. Nevertheless, it cannot be excluded that isoenzymes could be synthesized due to alternative splicing. In order to explain the presence of the lutein epoxide cycle in parasitic plants, it was suggested (Matsubara et al., 2003) that the epoxidase in these plants shows amino-acid sequence different from that in other plants and shows a broader substrate specificity. However, in the light of the present study it is difficult to accept this view. The amino-acid sequence similarity of the epoxidases within the Cuscuta genus is probably very high 
and their substrate specificity is expected to be also similar if not the same, but the capability for lutein epoxide synthesis among the species is clearly different. Based on the presently known gene sequences of zeaxanthin epoxidase the predicted amino-acid sequences for species of the same genus (Nicotiana plumbaginifolia and N. tabacum (Charron et al., 2005), Citrus limon AB114670, C. sinensis AB114662, and C. unshiu AB114654, Prunus mume AB253634 and $P$. armeniaca AF159948) are identical in the regions of the lipocalin $\beta$-barrel structure, three structurally conserved regions (I-III), as well as the ADP and FAD binding domains (Marin et al., 1996; Bugos et al., 1998; Charron et al., 2005), i.e. the regions most important for the catalytic activity. Moreover, it seems hardly probable that the very small differences in the structure of the ring on the side of the substrate molecule ( $\beta$ - and $\varepsilon$-ionone rings in zeaxanthin and lutein, respectively) opposite to the ring being oxidized would be critical for the enzyme activity. A possible reason for the different extent of lutein epoxide synthesis could be a varied accessibility of free lutein to the epoxidase, which probably requires the substrate in a protein-free form, similarly as the de-epoxidase (Yamamoto \& Bassi, 1996). In green leaves, lutein is specifically bound within LHC complexes and under such conditions no free lutein exists to be accessible for the epoxidase. This is probably also the case for a transgenic Arabidopsis lutOE line with an increased lutein synthesis (Matsubara et al., 2003), where the extra synthesized lutein is also firmly protein-bound. However, in organelles of non-green tissues (amyloplasts and etioplasts) where probably no specific lutein-binding proteins exist (expression of Lhcb genes is strongly light-dependent (Thompson \& White, 1991; Capel et al., 1998)), free lutein becomes available for the epoxidase and lutein epoxide is synthesized. Similar is probably the case also for some Cuscuta species where the photosynthetic apparatus is not well developed. This is indicated by the higher carotenoid to chlorophyll ratio (Table 1) than usually found in green leaves. The excess carotenoids (lutein) are probably not protein-bound and could be available for the epoxidase in the Cuscuta species showing a high lutein epoxide content.

Until the epoxidase is purified from Cuscuta species or any other plant and its substrate specificity determined, the question its substrate specificity remains open. Up to now, the only purified enzyme preparation available was that from red pepper cloned in Escherichia coli, which was specific for zeaxanthin only (Bouvier et al., 1996) in vitro, however, the cloned enzyme could behave differently than that isolated from natural sources. The latter task has not been accomplished yet despite many efforts in many laboratories, including ours. Moreover, ef- forts of cloning of the enzyme from other sources like Arabidopsis, in E. coli and Pichia pastoris have not been successful so far, either (Kruk, unpublished).

The presented results show that the investigated Cuscuta species differ considerably in the content of neoxanthin and the presence of the lutein epoxide cycle. Neoxanthin was not identified in three or four species while in others it was detected at a level reaching several percent of total xanthophylls. A relatively high content of lutein epoxide was found in three of the eight investigated species, while in others it was detected only at the low level which is often found in other higher plants. Our data also indicate that the presence of neoxanthin and of the lutein epoxide cycle is independent and variable among the Cuscuta species investigated. Moreover, the obtained results suggest that the capability for lutein epoxide synthesis probably depends not on the substrate specificity of the epoxidase but rather on the availability of lutein to the enzyme.

\section{Acknowledgements}

We would like to thank Professor Karin Krupinska from the University of Kiel (Germany) for the gift of Cuscuta species.

This work was supported by grant 2 P04A 06328 from the Ministry of Science, Poland.

\section{REFERENCES}

Berg S, Krause K, Krupinska K (2004) The rbcL genes of two Cuscuta species, C. gronovii and C. subinclusa, are transcribed by the nuclear-encoded plastid RNA polymerase (NEP). Planta 219: 541-546.

Bouvier F, d'Harlingue A, Hugueney P, Marin E, MarionPoll A, Camara B (1996) Xanthophyll biosynthesis - Cloning, expression, functional reconstitution, and regulation of $\beta$-cyclohexenyl carotenoid epoxidase from pepper (Capsicum annuum). J Biol Chem 271: 28861-28867.

Bugos RC, Hieber AD, Yamamoto HY (1998) Xanthophyll cycle enzymes are members of the lipocalin family, the first identified from plants. J Biol Chem 273: 1532115324.

Bungard RA, Ruban AV, Hibberd JM, Press MC, Horton P, Scholes JD (1999) Unusual carotenoid composition and a new type of xanthophyll cycle in plants. Proc Natl Acad Sci USA 96: 1135-1139.

Capel J, Jarillo JA, Madueno F, Jorquera MJ, Martinez-Zapater JM, Salinas J (1998) Low temperature regulates Arabidopsis Lhcb gene expression in a light-independent manner. Plant J 13: 411-418.

Charron JBF, Ouellet F, Pelletier M, Danyluk J, Chauve C, Sarhan F (2005) Identification, expression, and evolutionary analyses of plant lipocalins. Plant Physiol 139: 2017-2028.

Demming-Adams B, Adams WWIII (1992) Photoprotection and other responses of plants to high light stress. Annu Rev Plant Physiol Plant Mol Biol 43: 599-626. 
Garcia-Plazaola JI, Hernandez A, Errasti E, Becerril JM (2002) Occurrence and operation of the lutein epoxide cycle in Quercus species. Funct Plant Biol 29: 1075-1080.

Garcia-Plazaola JI, Hormaetxe K, Hernandez A, Olano JM, Becerril JM (2004) The lutein epoxide cycle in vegetative buds of woody plants. Funct Plant Biol 31: 815-823.

Garcia-Plazaola JI, Matsubara S, Osmond CB (2007) Review: The lutein epoxide cycle in higher plants: its relationships to other xanthophyll cycles and possible functions. Funct Plant Biol 34: 759-773.

Goodwin TW (1980) The Biochemistry of the Carotenoids, vol 1. Chapman and Hall, London, New York.

Grotz B, Molnar P, Stransky H, Hager A (1999) Substrate specificity and functional aspects of violaxanthin-deepoxidase, an enzyme of the xanthophyll cycle. J Plant Physiol 154: 437-446.

Grzyb J, Latowski D, Strzalka K (2006) Lipocalins - a family portrait. J Plant Physiol 163: 895-915.

Hibberd JM, Bungard RA, Press MC, Jeschke WD, Scholes JD, Quick WP (1998) Localization of photosynthetic metabolism in the parasitic angiosperm Cuscuta reflexa. Planta 205: 506-513.

Kruk J (2005) Occurrence of chlorophyll precursors in leaves of cabbage heads - the case of natural etiolation. J Photochem Photobiol B 80: 187-194.

Latowski D, Kruk J, Burda K, Skrzynecka-Jaskier M, Kostecka-Gugała A, Strzałka K (2002) Kinetics of violaxanthin de-epoxidation by violaxanthin de-epoxidase, a xanthophyll cycle enzyme, is regulated by membrane fluidity in model lipid bilayers. Eur J Biochem 269: 4656-4665.

Latowski D, Akerlund HE, Strzałka K (2004a) Violaxanthin de-epoxidase, the xanthophyll cycle enzyme, requires lipid inverted hexagonal structures for its activity. Biochemistry 43: 4417-4420.

Latowski D, Grzyb J, Strzalka K (2004b) The xanthophyll cycle - molecular mechanism and physiological significance. Acta Physiol Plant 26: 197-212.

Lichtenthaler HK (1987) Chlorophylls and carotenoids: pigments of photosynthetic biomembranes. Methods Enzymol 148: 350-382.

Marin E, Nussaume L, Quesada A, Gonneau M, Sotta B, Hugueney P, Frey A, Marion-Poll A (1996) Molecular identification of zeaxanthin epoxidase of Nicotiana plumbaginifolia, a gene involved in abscisic acid biosynthesis and corresponding to the ABA locus of Arabidopsis thaliana. EMBO J 15: 2331-2342.

Matsubara S, Gilmore AM, Osmond CB (2001) Diurnal and acclimatory responses of violaxanthin and lutein epox- ide in the Australian mistletoe Amyema miquelii. Aus I Plant Physiol 28: 792-800.

Matsubara S, Morosinotto T, Bassi R, Christian AL, FischerSchliebs E, Luttge U, Orthen B, Franco AC, Scarano FR, Forster B, Pogson BJ, Osmond CB (2003) Occurrence of the lutein-epoxide cycle in mistletoes of the Loranthaceae and Viscaceae. Planta 217: 868-879.

Matsubara S, Naumann M, Martin R, Nichol C, Rascher U, Morosinotto T, Bassi R, Osmond B (2005) Slowly reversible de-epoxidation of lutein-epoxide in deep shade leaves of a tropical tree legume may 'lock-in' luteinbased photoprotection during acclimation to strong light. J Exp Bot 56: 461-468.

Matsubara S, Morosinotto T, Osmond CB, Bassi R (2007) Short- and long-term operation of the lutein-epoxide cycle in light-harvesting antenna complexes. Plant Physiol 144: 926-941.

Schoefs B, Franck F (1988) Chlorophyll synthesis in darkgrown pine primary needles. Plant Physiol 118: 11591168.

Snyder AM, Clark BM, Robert B, Ruban AV, Bungard RA (2004) Carotenoid specificity of light-harvesting complex II binding sites - Occurrence of 9-cis-violaxanthin in the neoxanthin-binding site in the parasitic angiosperm Cuscuta reflexa. J Biol Chem 279: 5162-5168.

Snyder AM, Clark BM, Bungard RA (2005) Light-dependent conversion of carotenoids in the parasitic angiosperm Cuscuta reflexa L. Plant Cell Environ 28: 1326-1333.

Thompson WF, White MJ (1991) Physiological and molecular studies of light-regulated nuclear genes in higher plants. Annu Rev Plant Physiol Plant Mol Biol 42: 423466.

van der Kooij TAW, Krause K, Dorr I, Krupinska K (2000) Molecular, functional and ultrastructural characterisation of plastids from six species of the parasitic flowering plant genus Cuscuta. Planta 210: 701-707.

van der Kooij TAW, Krupinska K, Krause K (2005) Tocochromanol content and composition in different species of the parasitic flowering plant genus Cuscuta. J Plant Physiol 162: 777-781.

Yamamoto HY, Higashi RM (1978) Violaxanthin de-epoxidase. Lipid composition and substrate specificity. Arch Biochem Biophys 190: 514-522.

Yamamoto HY, Bassi R (1996) Carotenoids: localisation and function. In Oxygenic Photosynthesis: The Light Reactions. Ort DR, Yocum CF, eds, pp 539-563. Kluwer Academic Publishers, The Netherlands. 